\title{
BTA distance learning course in pulmonology
}

\author{
Irma Godoy ${ }^{1}$, Fernando Luiz Cavalcanti Lundgren²
}

The Brazilian Thoracic Association (BTA) statute states that one of the purposes of the BTA is to bring together physicians and other professionals involved in pulmonology research, teaching, and care, as well as to award specialist degrees in pulmonology and other areas of scientific expertise in accordance with its own policies and those of the Brazilian Medical Association. One of the many duties of the BTA board is to create departments, sections, and permanent or provisional committees aimed at developing research, teaching, care, continuing education, and standardization in areas of expertise and related areas, as well as to approve publication of guidelines and recommendations made by these organizations.

The duties of the director of education include the following: to coordinate, together with the scientific director, all BTA courses; to coordinate the development of the examination required for a specialist degree in pulmonology and the renewal thereof; to foster education in pulmonology by focusing on its promotion, quality, and continued improvement; and to represent the BTA with educational regulatory bodies.

The BTA is committed to establishing standards of excellence in respiratory care through education and professional development activities. The BTA currently offers a range of activities through its live and webbased continuing education programs, virtual learning environment, and nationwide pulmonology-related events in several areas.

The training of future pulmonologists is an essential part of all BTA education activities. Data show that, in Brazil, there are currently 66 medical residency programs in pulmonology, with 124 openings for first-year residents. The pulmonology program was last updated with the Brazilian National Medical Residency Committee in 2006. A new proposal was sent in 2011 and has yet to be approved. There are graduate programs in pulmonology that are accredited by the BTA.

Not all institutions providing education in pulmonology offer complete training in this field, which is, unfortunately, not widely available, as well as being costly to recently graduated physicians. In addition, referral centers for specific conditions are scattered throughout the country, and, given the size of the country, access can be difficult. Therefore, there is a clear need to harmonize and improve pulmonology training in Brazil.

To contribute to reducing the differences in pulmonology training, the BTA has created its first distance learning program, the "BTA distance learning course in pulmonology". For 2017, a total of 15 lectures will be recorded and made available on a distance learning platform. The course will be available on the BTA website as of July 1, 2017 and will cover the following topics:

- What are the applications of pulmonary function tests with lung volume and DLCO measurements and how should the results be interpreted?

- What are the applications of ergospirometry and how should the results be interpreted?

- Understanding polysomnography reports

- Long-term home oxygen therapy: current status and use in special conditions

- How should a patient whose echocardiographic report suggests the presence of pulmonary hypertension be investigated?

- How should the results of right heart catheterization be interpreted?

- How should asthma control be assessed in clinical practice?

- Inflammatory markers of asthma in clinical practice

- What are the health consequences of using tobacco products and other smoking products (i.e., electronic cigarettes, water pipes, and marijuana)?

- How should pulmonary nodules be approached and monitored?

- Lung cancer biomarkers

- Home noninvasive ventilation (indications, management, and costs)

- Diagnostic approach to and management of adult patients with cystic fibrosis

- Indications for the GeneXpert test and interpretation of its results in the diagnosis of tuberculosis

- Diagnostic and therapeutic management of the various forms of acute and chronic aspergillosis

All of the professors who were invited to give lectures accepted to do so at no cost and are well-known experts in the field. They will answer any questions posted by participants within 15 days after the contents have been published on the distance learning platform. At the end of each lecture, participants must answer self-assessment questions.

Instructions on how to participate in the course:

1. In order to participate, residents and graduate students in pulmonology enrolled in programs accredited by the BTA, as well as BTA members, must enroll in the course. All relevant information is available on the distance learning platform.

2. Over a period of 15 days after the lectures have been made available on the website, lecturers will answer the questions posted on the platform.

3. All participants must answer self-assessment questions; those who answer at least $70 \%$ of the questions correctly will be certified. 
4. All BTA members are invited to watch the lectures; however, only residents and graduate students enrolled in programs accredited by the BTA are allowed to interact with the lecturers.

5. Participants are allowed to watch the lectures and answer the questions three times.
The BTA is confident that participation will be high and that the course will be of value to future pulmonologists. We thank all participating professors and students in advance, whose comments and suggestions for future modules will be most welcome. 